QUANTUM PROBABILITY

BANACH CENTER PUBLICATIONS, VOLUME 73

INSTITUTE OF MATHEMATICS

POLISH ACADEMY OF SCIENCES

WARSZAWA 2006

\title{
AXIOMS FOR QUANTUM PROBABILITY
}

\author{
LUIGI ACCARDI \\ Centro V. Volterra \\ Università di Roma Tor Vergata \\ E-mail: accardi@volterra.uniroma2.it \\ Web: http://volterra.uniroma2.it
}

The date of birth of the "new" quantum mechanics (as opposed to the old semiclassical theory) is usually identified with the publication of Heisenberg's paper where the commutation relations were first introduced (1925). One year after it became clear, with the emergence of the statistical interpretation, that this theory was as much a new mechanics as a new probability calculus.

This discovery caused a change of perspective on the 6 -th of the twenty-three problems stated by Hilbert in 1900, i.e. twenty five years before, at the Paris International Congress of Mathematicians, namely:

... to treat axiomatically those physical disciplines in which already today mathematics plays a predominant role... these are in the first place the calculus of probability and mechanics...

The key point of this new perspective was that, thanks to it, the motivation of the proposed axiomatization was no longer a matter of aesthetics and of systematization, but the dramatic need to substantiate the new mathematical formalism with a new physical intuition. This formalism worked remarkably well: it was "a chicken laying golden eggs" and most physicists were busy in collecting and using them but a few far looking scientists also wanted to understand where these golden eggs came from. As remarked by Heisenberg himself in one of his most quoted papers [Hei27] (where the indeterminacy principle was first introduced), the new mathematical formalism was far from intuition: why do we need complex wave functions to describe probabilities? why Hilbert spaces? where do the commutation or anti-commutation relations come from? For several decades nobody knew the answers to these questions.

People looked for an axiomatization of the theory on the basis of the following reasoning: if the main mathematical features of quantum theory did not come out by chance,

2000 Mathematics Subject Classification: 60H40.

The paper is in final form and no version of it will be published elsewhere. 
but (as everybody was intimately convinced) they are an expression of new physical requirements, then the only way to acquire a really satisfactory understanding of these requirements is to produce a list of them which is exhaustive, in the sense that from it all the new mathematical features of the theory can be deduced. Thus, paradoxically, the research for an axiomatic foundation of quantum theory was not conceived as a systematization of an established intuition, but as an heuristic tool to create a new one.

It took however more than 50 years to fully realize the implications of this change of perspectives, and a posteriori we can understand the reasons of this delay: before attempting a comparison between the quantum and the classical probabilistic models, it was necessary to elucidate the mathematical structure of the new quantum theory independently of its physical implications. In this enterprise a crucial role was played by mathematicians such as von Neumann, Weyl, ... and physicists such as Dirac, Pauli, Majorana, Wigner, Fock, .... From the mathematical side this process was initiated by Hilbert himself in a joint paper with von Neumann and Nordheim [HvNN27] in 1927 (remember that the first Heisenberg paper on quantum mechanics is of 1925). In the introduction to this paper the authors specify what they mean when proposing ... to treat axiomatically a physical discipline in the following way:

... to formulate the physical requirements so completely that the mathematical formalism becomes uniquely determined by them ...

This distinction between the physical requirements on one side and the mathematical formalism on the other should be clearly kept in mind by anybody who shares with these great men of the past the curiosity for the clarification of the conceptual and foundational aspects of science: a good axiomatization should in some sense prove the intrinsic necessity of a certain mathematical formalism.

Clearly the first step in such a proof is a precise description of this formalism. The second step is its deduction from physical requirements.

For classical probability the first step was realized by Kolmogorov in his 1933 monograph Grundbegriffe der Wahrscheinlichkeitsrechnung [Kolmo33]. It is a common saying that this monograph contains an "axiomatization" of classical probability theory. However it should be made clear that the term "axiomatization" in this sentence should be meant as "description" of the mathematical model and not in the sense of its deduction.

It should also be noted that Kolmogorov's monograph [Kolmo33] came to light in a time when the most advanced physical theory, i.e. quantum theory, was already making extensive use of a completely different mathematical formalism to model probabilistic phenomena.

The challenge posed to all probabilists by this new probability calculus was pointed out in Feynman's communication at the 2-nd Berkeley Symposium on Probability and Statistics [Feyn51].

In addition to the above quoted mathematicians, further contributions to our understanding of the foundational problems of quantum theory came from Segal [Segal47], who laid the foundations of the algebraic approach to quantum mechanics, later developed by Haag and Kastler [HaaKa64] which, in its turn, greatly stimulated the development 
of operator theory, and from Mackey whose monograph [Mackey76], based on a earlier proposal due to Birkhoff and von Neumann [BirkvonN36], had a vast influence on the physical literature on the foundations giving rise to a line of research known under the name of "Quantum Logic". This line of research produced two important mathematical results, namely the Gleason theorem [Gleas57], [Paszk85], and an infinite dimensional extension of the coordinatization theorem of projective geometry (cf. [Varadar68] for a survey). However quantum logic failed in its main objective of solving the problem posed by Hilbert, von Neumann and Nordheim because it did not succeed in finding a satisfactory solution to the following problems:

(i) To find a convincing physical justification of the "covering axiom", needed to distinguish between the lattice of all projections of a Hilbert space and the huge, and not easily controllable, class of all non-distributive lattices.

(ii) To give a physically meaningful interpretation of the two basic operations of quantum logic, namely the "meet" and "join" of two non-commuting projections (cf. the discussion in [Jau68], [Piron76]).

(iii) To find a convincing physical justification of the emergence of complex, rather than real Hilbert spaces.

Since the goal of this line of research is exactly to deduce the complex Hilbert space structure from physically meaningful axioms, it is unclear to many people why the replacement of this axiom by other mathematical postulates whose physical meaning is as unclear as the original one, should be considered a satisfactory solution to the problem.

In the early 1980's a solution of these problems emerged in the context of quantum probability. In fact one could say that the converse statement better reflects the historical development: the new ideas, emerged from the developments related to the sixth Hilbert problem, lead to the conviction that what was at stake in these developments was not merely a formal non-commutative extension of the mathematical model of classical probability, but a conceptual revolution in our way of understanding the laws of chance, comparable only to what happened in the first half of the XIX-th century with geometry, in the end of the same century with functional analysis and in the beginning of the XX-th century with logic.

The key steps in this conviction were:

(i) the identification of a common root in all the so-called quantum paradoxes;

(ii) the discovery of the possibility of giving an experimental proof of the necessity of non-Kolmogorovian probabilistic models (statistical invariants).

(iii) the discovery that the statistical invariant technique was so fine to allow experimental distinction between the choice of real or complex Hilbert space.

(iv) the formulation of a set of axioms for probability theory which allows the mathematical deduction of the whole mathematical model of quantum mechanics from a (model independent) reformulation of the Heisenberg uncertainty principle.

In the following I will quickly review the mathematical aspects of these developments. The main emphasis of this exposition will not be on proofs (for these one can look at [Ac82c] or [Ac95a]) but on the global picture and on the questions which are still open. 
These are not few and not trivial, but the general deductive path from the physical requirements to the Hilbert space structure is clearly outlined.

The perspectives opened to probability theory by these developments are so wide and exciting as those opened to geometry by the discoveries which took place in the mid XIX-th century: the experiments compel us to go beyond the Kolmogorov model; the axioms show that the qualitative statement of the existence of incompatible observables mysteriously encrypts the Hilbert space structure and that the experiments determine the choice of the number field; the deep understanding of the hidden axiom of probability theory (Bayes' definition of conditional probability, not discussed here) allows to understand the probabilistic roots of the so-called "quantum paradoxes" and to clarify them both from the mathematical and the intuitive point of view; it also gives us a clue to look for non Kolmogorovian models outside quantum theory. In some sense we can claim that all branches of mathematics can be made non-commutative, but probability must.

1. Axioms of probability. In this section we list a set of axioms which account for the intuitive properties of measurements. The resulting structure is very general and includes both the classical and the quantum case.

Let $\mathcal{M}$ be a set whose elements we call (1-st kind) measurements, or simply measurement apparata, or apparata or instruments. On this set we introduce the following axioms.

(A1) Composition axiom. There exists a binary composition law

$$
(X, Y) \in \mathcal{M} \times \mathcal{M} \Longrightarrow X \cdot Y \in \mathcal{M}
$$

called multiplication.

Interpretation: The multiplication of two instruments $X, Y$ corresponds to the consecutive performance of each of them (in series):

$$
\text { input } \Longrightarrow X \Longrightarrow Y \Longrightarrow \text { output. }
$$

\section{(A2) Associativity of the multiplication.}

$$
(X \cdot Y) \cdot Z=X \cdot(Y \cdot Z)
$$

Interpretation: The definition of instrument is largely arbitrary: one can always consider two consecutive measurements as a single one according to the following scheme:

$$
\Longrightarrow(X \Longrightarrow Y) \Longrightarrow Z \Longrightarrow X \Longrightarrow(Y \Longrightarrow Z) \Longrightarrow \text {. }
$$

(A3) Existence of the "all-pass"-measurement. There exists a measurement, denoted 1, characterized by the property:

$$
X \cdot 1=1 \cdot X=X, \quad \forall X \in \mathcal{M} .
$$

Interpretation: 1 is the trivial measurement in which there is no interaction between the system and the apparatus therefore every system passes through the apparatus.

(A4) Existence of the "no-pass"-measurement. There exists a measurement, denoted 0 , characterized by the property:

$$
X \cdot 0=0 \cdot X=0, \quad \forall X \in \mathcal{M} .
$$


Interpretation: 0 is the trivial measurement which destroys every system, therefore no system passes through the apparatus.

(A5) Existence of the time reversal operation. There exists an operation

$$
*: \mathcal{M} \Longrightarrow \mathcal{M}
$$

called time reversal, such that

$$
\begin{gathered}
(X \cdot Y)^{*}=Y^{*} X^{*}, \\
\left(X^{*}\right)^{*}=X .
\end{gathered}
$$

Moreover

$$
1^{*}=1, \quad 0^{*}=0 .
$$

Interpretation: The time reversal of $X$ corresponds to doing in reverse order all the physical operations corresponding to the measurement $X$.

$$
(\Longrightarrow X \Longrightarrow)^{*} \quad=\quad \Longleftarrow X^{*} \Longleftarrow
$$

A symmetric instrument is one in which the order of the sequential operations is irrelevant:

$$
X^{*}=X \text {. }
$$

(A6) Randomization axiom. For each instrument $X$ and each number $p \in[0,1]$, there exists an instrument, denoted $p X$, with the following properties:

$$
\begin{gathered}
(p X) \cdot Y=p(X \cdot Y)=X \cdot(p Y), \\
(1 \cdot X)=X, \quad(0 \cdot X)=0,
\end{gathered}
$$

Interpretation: For any input, $p X$ either produces the same output as $X$ or no output. In many trials of $p X$ with the same preparation $X$ the ratio

$$
\frac{\text { \#outputs of } p X}{\text { \#outputs of } X}
$$

is approximatively $p$.

Definition 1. Two instruments $X, Y$ are called compatible if:

$$
X \cdot Y=Y \cdot X \text {. }
$$

Interpretation: the information in the two experiments is cumulative in the sense that the performance of any of the two experiments after the other one does not destroy the information gained in the previous experiment:

$$
\Rightarrow X \Rightarrow Y \Rightarrow \quad=\quad \Rightarrow Y \Rightarrow X \Rightarrow \text {. }
$$

The previous axioms concerned measurements in series. Now we discuss measurements in parallel. Because of the indeterminacy principle, not all measurements can be performed in parallel. This means that the corresponding composition law cannot be everywhere defined.

(A7) Sum axiom. There exists a binary composition law among compatible measurements

$$
X \cdot Y=Y \cdot X \Rightarrow X+Y \in \mathcal{M}
$$

satisfying the following conditions: 


\section{(A7.1) Commutativity.}

$$
X+Y=Y+X
$$

(A7.2) Associativity.

$$
(X+Y)+Z=X+(Y+Z)
$$

(A7.3) Neutrality of zero.

$$
X+0=0+X=X
$$

(A7.4) Cancellation law.

$$
X+Y=X+Z \Rightarrow Y=Z \text {. }
$$

(A7.5) Distributivity. If $X, Y, Z$ are pairwise compatible, then

$$
(X+Y) \cdot Z=X \cdot Z+Y \cdot Z \text {. }
$$

Interpretation: when restricted to compatible observables, the theory becomes entirely classical and the above operations are standard operations on the results of measurements.

The interpretation of the axioms is further discussed in [Ac95a].

DEFINITION 2. An algebra of measurements is a quintuple:

$$
\{\mathcal{M}, \cdot,+, * \text {, multiplication by } p \in[0,1]\}
$$

where $\mathcal{M}$ is a set and the operations $\cdot,+, *$ and the multiplication by $p \in[0,1]$ satisfy the Axioms (A1), .., (A7).

Definition 3. An algebra of measurements $\mathcal{M}$ is called representable if there exists a real $*$ algebra $\mathcal{A}$ (by this we mean associative $*$-algebra over the reals with an identity) and an injective map $j: \mathcal{M} \Rightarrow \mathcal{A}$ which preserves the algebraic structure, i.e. such that

$$
\begin{gathered}
j(X)^{*}=j(X)^{*}, \\
j(X Y)=j(X) \cdot j(Y), \\
j(p X)=p j(X), \quad \forall p \in[0,1], \\
j(1)=1_{\mathcal{A}}, \quad j(0)=0, \\
j(X+Y)=j(X)+j(Y) \quad \text { if } \quad X Y=Y X .
\end{gathered}
$$

Moreover it is required that $\mathcal{A}$ is minimal among the algebras with this property, i.e. that if $\mathcal{B}$ is a $*$-algebra and $k: \mathcal{M} \Rightarrow \mathcal{B}$ is a map satisfying the above identities, then there exists a $*$-homomorphism $\alpha: \mathcal{A} \Rightarrow \mathcal{B}$ such that:

$$
k=\alpha \circ j,
$$

The pair $\{\mathcal{A}, j\}$ is called the $*$-algebra generated by $\mathcal{M}$.

Proposition 1. If $\mathcal{M}$ is representable, then the real $*$-algebra $\mathcal{A}$ is determined by $\mathcal{M}$ up to isomorphism.

In the following we shall restrict our attention to representable measurement algebras. and we shall use the same term measurement algebra for the associated real $*$-algebra $\mathcal{A}$.

Open Problem: Is every algebra of measurements $\mathcal{M}$ representable? 
From now on $\mathcal{A}$ is a *-algebra.

Definition 4. An algebra of measurements $\mathcal{M}$ is called classical if it is representable and the associated real *-algebra $\mathcal{A}$ is commutative.

2. Quantum axioms: the Heisenberg principle. In this section we formulate in terms of measurements the fundamental new qualitative feature of quantum physics: the Heisenberg principle. The following statement can be considered as a model independent formulation of this principle: there exist incompatible observables.

Equivalently: the $*$-algebra of measurements is non-commutative. Notice that this formulation is universal, i.e. not restricted to the domain of micro-physics. To formulate this idea precisely, we need to introduce the notion of an observable.

Definition 5. A partition of the identity in $\mathcal{A}$ is a set $A=\left\{A_{1}, \ldots, A_{n}\right\}$ of elements of $\mathcal{A}$ satisfying the following relations:

$$
\begin{gathered}
A_{j}=A_{j}^{2}, \\
A_{j} \cdot A_{k}=\delta_{j k} A_{k}, \quad j=1, \ldots, n, \\
\sum_{j=1}^{n} A_{j}=1, \\
A_{j}=A_{j}^{*} .
\end{gathered}
$$

Elements of a partition of the identity are also called filters.

Denote:

$$
\kappa:=\operatorname{center}(\mathcal{A})
$$

and, for any partition of the identity $A=\left\{A_{1}, \ldots, A_{n}\right\}$, in $\mathcal{A}$, denote:

$$
\mathcal{A}_{A}:=\left\{\sum_{j} \lambda_{j} A_{j}: \lambda_{1}, \ldots, \lambda_{n} \in \kappa\right\} .
$$

Definition 6. A partition of the identity is called maximal if the algebra $\mathcal{A}_{A}$ coincides with its commutant, i.e., if for any $X$ in $\mathcal{A}$,

$$
X A_{j}=A_{j} X \quad \forall j=1, \ldots, n \Longleftrightarrow X \in \mathcal{A}_{A} .
$$

Definition 7. An observable is a symmetric instrument (i.e. self-adjoint element of $\mathcal{A}$ ) of the form

$$
A:=\sum_{\alpha} a_{\alpha} A_{\alpha}
$$

where $\left(a_{\alpha}\right)$ are real numbers and $A_{\alpha}$ is a partition of the identity.

$A$ is called maximal if $\left(A_{\alpha}\right)$ is maximal. Observables in the center $\kappa$ are called superselection observables.

(A8) Heisenberg principle (generic form). There exist two different maximal partitions of the identity $\left(A_{\alpha}\right),\left(B_{\beta}\right)$, i.e. for at least one pair of indices $\alpha, \beta$ one has:

$$
A_{\alpha} B_{\beta} \neq 0 \text {. }
$$


REMARK 1. In the classical case, there can be at most one maximal partition of the identity, and conversely, if this is the case, then the measurement algebra is classical in the sense of the previous definition.

The algebra of projections generated by an abelian measurement algebra is Boolean and Stone's representation theorem provides a standard mathematical model for the Boolean algebras in terms of subsets of some set $\Omega$. This explains the classical probabilistic model of events as subsets of a given set.

\section{Quick review of terminology:}

- apparatus $\equiv$ element of $\mathcal{A}$

- concatenation of apparata $\equiv$ multiplication

- simultaneous action of compatible apparata $\equiv$ addition

- filter $\equiv$ projection

- elementary filter $\equiv$ atomic projection (precise value of an observable)

- the output of an elementary filter is at most one value at the time $\equiv \alpha \neq \beta \Rightarrow$ $A_{\alpha} A_{\beta}=0$

- the output of a maximal set of elementary filters (in parallel) exhausts all possibilities $\equiv \sum_{\alpha} A_{\alpha}=1$

- equivalence class of [maximal] observables $\equiv$ [maximal] partition of the identity ( $A \equiv B \Leftrightarrow A$ and $B$ correspond to the same partition of the identity and there is a one-to-one correspondence between the values of $A$ and those of $B$ ).

- superselection observables $\equiv$ self-adjoint elements of the center of $\mathcal{A}$.

\section{Schwinger algebras}

Definition 8. A [generic] Schwinger algebra of rank $n$ over a set $T$ is a triple

$$
\left\{\mathcal{A}, T,(A(x))_{x \in T}\right\}
$$

where $\mathcal{A}$ is a real associative $*$-algebra, $T$ is a set and, for every $x$ in $T$,

$$
A(x)=\left\{A_{1}(x), \ldots, A_{n}(x)\right\}
$$

is a maximal partition of the identity in $\mathcal{A}$ such that for any $x, y$ in $T$, for any $j, k=$ $1, \ldots, n$ and for any $\gamma \in \kappa$, the following (genericity) conditions hold:

$$
\begin{gathered}
\gamma A_{j}(x) A_{k}(y)=0 \Longleftrightarrow \gamma=0, \\
\gamma A_{j}(x) \geq 0 \Longleftrightarrow \gamma \geq 0 .
\end{gathered}
$$

The sub-algebra of $\mathcal{A}$ generated over the center $\kappa$ by the partition of the identity $\left\{A_{1}(x), \ldots, A_{n}(x)\right\}$ will be denoted by $\mathcal{A}(x)(x \in T)$.

Writing $A_{j}(x)$ as $A_{j}(x) \cdot A_{j}(x)$, it follows from (4) that equality holds on the right hand side of (5) if and only if $\gamma=0$.

DeFinition 9 . Let $\kappa$ be a real $*$-algebra and let $n$ be an integer or $+\infty$. An $n$-dimensional $\kappa$-valued stochastic matrix is a matrix $P=\left(p_{i j}\right)(i, j=1, \ldots, n)$ such that

$$
p_{i j} \in \kappa, \quad p_{i j} \geq 0, \quad \sum_{j=1}^{n} p_{i j}=1
$$


where 1 denotes the identity in $\kappa$. If each $p_{a b}$ is invertible, we write

$$
p_{a b}>0 \text {. }
$$

If also the condition

$$
\sum_{i=1}^{n} p_{i j}=1
$$

is satisfied, then we say that $P$ is a $\kappa$-valued bi-stochastic matrix.

Recall that, by definition, the set of positive elements of a $*$-algebra $\mathcal{A}$ is the cone (closed if there is a topology) generated by the elements of the form $a^{*} a$ with $a \in \mathcal{A}$.

The following theorem shows that Schwinger algebras are canonically associated to bi-stochastic matrices over their center.

TheOREM 1. Let $\left\{\mathcal{A}, T,(A(x))_{x \in T}\right\}$ be a Schwinger algebra of rank $n$. Then for any pair of elements $x, y$ of $T$ there exists a $\kappa$-valued $n$-dimensional bi-stochastic matrix $P=\left(p_{i j}\right)$ $(i, j=1, \ldots, n)$, such that for any $i, j=1, \ldots, n$ one has:

$$
\begin{gathered}
A_{i}(x) A_{j}(y) A_{i}(x)=p_{i j}(x, y) A_{i}(x), \\
p_{i j}(x, y)=p_{j i}(y, x) .
\end{gathered}
$$

Proof. We include the proof because it is very simple and shows that this conceptually important result follows easily and naturally from qualitative axioms.

$A_{i}(x) A_{j}(y) A_{i}(x)$ is in the commutant of $\mathcal{A}(x)$, hence in $\mathcal{A}(x)$ by maximality. The mutual orthogonality of the $A_{i}(x)$ implies (8) for some $p_{i j}(x, y) \in \kappa$. Positivity follows from (5) and

$$
A_{i}(x) A_{j}(y) A_{i}(x)=\left[A_{j}(y) A_{i}(x)\right]^{*}\left[A_{j}(y) A_{i}(x)\right] .
$$

Normalization follows from (4) and $\sum_{j} A_{j}(y)=1$. Finally (9) follows from (4) and associativity:

$$
\left[A_{i}(x) A_{j}(y) A_{i}(x)\right] A_{j}(y)=A_{i}(x)\left[A_{j}(y) A_{i}(x) A_{j}(y)\right]
$$

REMARK 2. Not only two maximal observables in a Schwinger algebra canonically define a transition probability matrix, but this matrix has necessarily the symmetry property (9) which is true in the usual Hilbert space model due to (using self-explanatory notations):

$$
p_{i j}(x, y)=\left|\left\langle\psi_{i}(x), \phi_{j}(y)\right\rangle\right|^{2}=\left|\left\langle\phi_{j}(y), \psi_{i}(x)\right\rangle\right|^{2}=p_{j i}(y, x)
$$

Statement of the compatibility problem. We have shown that every Schwinger algebra

$$
\left\{A, T,(A(x))_{x \in T}, \kappa\right\}
$$

defines a correspondence

$$
(A(x), A(y)) \mapsto P(x, y)=\left(p_{i j}(x, y)\right)
$$

between pairs of maximal observables and $\kappa$-valued bi-stochastic matrices, characterized by the properties:

$$
\begin{gathered}
A_{i}(x) \cdot A_{j}(y) \cdot A_{i}(x)=p_{i j}(x, y) \cdot A_{i}(x), \\
p_{i j}(x, y)=p_{j i}(y, x) .
\end{gathered}
$$


The converse statement is the compatibility problem, i.e. given:

- a set $T$

- a family $\{P(x, y): x, y \in T\}$ of $n \times n \kappa$-valued bi-stochastic matrices, determine under which conditions there exists a Schwinger algebra of rank $n$

$$
\left\{A, T,(A(x))_{x \in T}\right\}
$$

such that $\forall x, y \in T$ and $\forall i, j=1, \ldots, n$,

$$
A_{i}(x) A_{j}(y) A_{i}(x)=p_{i j}(x, y) A_{i}(x) .
$$

We know that the symmetry condition

$$
p_{a b}(x, y)=p_{b a}(y, x)
$$

is a necessary condition for the solution of the problem. We also know that

$$
P(x, x)=1, \quad \forall x \in T .
$$

Moreover the genericity conditions (4) and (5) imply that:

$$
p_{a b}(x, y)>0, \quad \forall x \in T \quad \forall a, b=1, \ldots, n .
$$

Physical interpretation: given the physical data, i.e.

(i) a family $\{\bar{A}(x): x \in T\}$ of $n$-valued maximal observables and their values $a_{j}(x)$ $(j=1, \ldots, n)$

(ii) the (experimentally measurable) transition probabilities $p_{i j}(x, y)$ among these values:

$$
\operatorname{Prob}\left\{\bar{A}(y)=a_{j}(y) \mid \bar{A}(x)=a_{i}(x)\right\}=p_{i j}(x, y)
$$

one looks for conditions under which a Schwinger algebra for these data exists.

REMARK 3. The compatibility problem is the quantum analogue of the following problem in classical probability: given a family of transition probability matrices

$$
\{P(s, t): s<t, s, t \in \mathbb{R}\}
$$

(i.e. in this case $T=\mathbb{R}_{+}$), when does there exist a Markov process $(A(t))$ such that for each $s<t$, the transition matrix canonically associated to the pair of random variables $A(s), A(t)$ is $P(s, t)$ ?

It is known that the classical compatibility problem has a positive solution if and only if the family of transition probability matrices $(P(s, t))$ satisfies the ChapmanKolmogorov equation:

$$
P(r, s) \cdot P(s, t)=P(r, t), \quad r<s<t .
$$

In other words: the Chapman-Kolmogorov equation is a compatibility condition for a family $(P(s, t))$ of stochastic matrices to admit a classical Markov model.

We will now prove that the Schrödinger equation is the solution of the analogue compatibility problem for Schwinger algebras. 
4. Heisenberg algebras: deduction of the superposition principle. In this section we prove an analogue, for Schwinger algebras, of Stone's representation theorem. This will follow from the solution of the compatibility problem for a particular class of Schwinger algebras: the so-called Heisenberg algebras. The following lemma motivates this notion.

Lemma 1. Let $\left\{\mathcal{A}, T,(A(x))_{x \in T}\right\}$ be a Schwinger algebra. For any pair of elements $x, y$ of $T$ the set

$$
\left\{A_{i}(x) A_{j}(y): i, j=1, \ldots, n\right\}
$$

is linearly independent over $\kappa$.

Definition 10. In the above notations, the algebra $\mathcal{A}$ is called a Heisenberg algebra if, for any pair of elements $x, y$ of $T$, the set

$$
\left\{A_{i}(x) A_{j}(y): i, j=1, \ldots, n\right\}
$$

is a $\kappa$-basis of $\mathcal{A}$.

In other words: Heisenberg algebras are the smallest possible Schwinger algebras.

Theorem 2. Let $\mathcal{A}$ be a Heisenberg algebra and let $A=\left(A_{a}\right)$ and $B=\left(B_{b}\right)$ be a generic pair of maximal partitions of the identity. Then there exist elements $\gamma_{a b}^{c d} \in \kappa(a, b, c, d=$ $1, \ldots, n)$, such that

$$
B_{b} \cdot A_{a}=\sum_{c, d=1}^{n} \gamma_{a b}^{c d} A_{c} \cdot B_{d}
$$

these elements will be called the structure constants of $\mathcal{A}$ in the $\left(A_{a} B_{b}\right)$-basis.

The following theorem shows that, in analogy with the theory of Lie algebras, the structure constants of a Schwinger algebra encode all the information on the algebra

Theorem 3. Let $\mathcal{A}$ be a unital associative algebra and let $\kappa$ denote its center. Let $\left(A_{a}\right)$, $\left(B_{b}\right)(a, b=1, \ldots, n)$ be partitions of the identity in $\mathcal{A}$ such that the set $\left\{A_{a} \cdot B_{b}: a, b=\right.$ $1, \ldots, n\}$ is a $\kappa$-basis of $\mathcal{A}$, and let $\gamma_{a b}^{c d}(a, b=1, \ldots, n)$ be elements of $\kappa$ such that the identity (15) holds. Then

$$
\begin{gathered}
\sum_{a=1}^{n} \gamma_{a b}^{a^{\prime} b^{\prime}}=\delta_{b b^{\prime}}, \\
\sum_{b=1}^{n} \gamma_{a b}^{a^{\prime} b^{\prime}}=\delta_{a a^{\prime}}, \\
\gamma_{a^{\prime} b}^{a b^{\prime}} \gamma_{a^{\prime \prime} b^{\prime}}^{a b^{\prime \prime}}=\gamma_{a^{\prime} b}^{a b^{\prime \prime}} \cdot \gamma_{a^{\prime \prime} b^{\prime}}^{a^{\prime} b^{\prime \prime}}
\end{gathered}
$$

If, moreover, $\mathcal{A}$ is a *-algebra, then

$$
\begin{gathered}
\sum_{c, d=1}^{n}\left(\gamma_{a b}^{c d}\right)^{*} \cdot \gamma_{c d}^{c^{\prime} d^{\prime}}=\delta_{a c^{\prime}} \cdot \delta_{b d^{\prime}} \\
\left(\gamma_{a^{\prime} b}^{a b^{\prime}}\right)^{*} \cdot \gamma_{a b^{\prime}}^{a^{\prime \prime} b^{\prime \prime}}=\sum_{e, d=1}^{n} \gamma_{a^{\prime} b^{\prime}}^{a^{\prime \prime} d} \cdot \gamma_{e d}^{a^{\prime \prime} b^{\prime \prime}} \cdot \gamma_{a b}^{e b^{\prime \prime}} .
\end{gathered}
$$


Conversely, if $\kappa$ is a unital commutative associative real $*$-algebra and $\gamma_{a b}^{c d}(a, b=1, \ldots, n)$ are elements of $\kappa$ satisfying (16), (17), (18), then there exist an associative algebra $\mathcal{A}$ with center $\kappa$ and two partitions of the identity in $\mathcal{A}, A=\left(A_{a}\right), B=\left(B_{b}\right)$, such that $A_{a} \cdot B_{b}$ is a basis of $\mathcal{A}$ over $\kappa$ and (15) holds. If, moreover, (19) and (20) hold, then $\mathcal{A}$ has a unique structure of a *-algebra whose involution is characterized by the property that its restriction to $\kappa$ coincides with the original involution on $\kappa$, and for all $a, b$,

$$
A_{a}=A_{a}^{*}, \quad B_{b}=B_{b}^{*} .
$$

\section{The case of two maximal observables: emergence of the Hilbert space}

THEOREM 4. Let $\mathcal{A}$ be an associative real algebra generated by the maximal partitions of the identity $A=\left(A_{a}\right) ; B=\left(B_{b}\right)$. Assume that the transition probability matrix $P=\left(p_{a b}\right)$ between $A$ and $B$ is strictly positive in the sense of (6) and denote by $\gamma_{a b}^{c d}$ the structure constants of $\mathcal{A}$ in the $\left(A_{a} B_{b}\right)$-basis. Then there exists a $\kappa$-valued matrix $U=\left(u_{a b}\right)$ such that

$$
\begin{gathered}
p_{a b}=\left|u_{a b}\right|^{2}:=u_{a b}^{*} u_{a b}, \quad a, b=1, \ldots, n, \\
\sum_{b=1}^{n} u_{a^{\prime} b} u_{a b}^{*}=\delta_{a, a^{\prime}}, \quad a, a^{\prime}=1, \ldots, n, \\
\sum_{a=1}^{n} u_{a b}^{*} u_{a b^{\prime}}=\delta_{b b^{\prime}}, \quad b, b^{\prime}=1, \ldots, n, \\
\gamma_{a b}^{a^{\prime} b^{\prime}}=\frac{u_{a b^{\prime}} u_{a^{\prime} b}}{u_{a b} u_{a^{\prime} b^{\prime}}} p_{a b}, \quad a, b, a^{\prime}, b^{\prime}=1, \ldots, n .
\end{gathered}
$$

Conversely, if $\kappa$ is a real commutative *-algebra, then, given a strictly positive $\kappa$-valued strictly positive bi-stochastic matrix $P=\left(p_{a b}\right)$, and a $\kappa$-valued matrix $U=\left(u_{a b}\right)$ satisfying (23), (24), (25), there exist:

- an associative real algebra $\mathcal{A}$ with center $\kappa$,

- two maximal partitions of the identity $A=\left(A_{a}\right) B=\left(B_{b}\right)$ in $\mathcal{A}$ with transition matrix $P$ such that the $\gamma_{a b}^{c d}$, defined by the right hand side of (25), are the structure constants of $\mathcal{A}$ in the $\left(A_{a} B_{b}\right)$-basis.

\section{Arbitrary number of observables: deduction of the Schrödinger equation}

THEOREM 5. For a family of transition probability matrices $\{P(x, y): x, y \in T\}$ satisfying the conditions listed above the following assertions are equivalent:

(i) There exists a Heisenberg algebra $\left\{\mathcal{A}, T,(A(x))_{x \in T}\right\}$ with center $\kappa$, such that for each $x, y \in T, P(x, y)$ is the transition probability matrix canonically associated to the pair $(A(x), A(y))$.

(ii) For each $x, y \in T$ there exists a $\kappa$-valued unitary matrix $U(x, y)=\left(u_{i j}(x, y)\right)$ such that for each $x, y, z \in T, i, j=1, \ldots, n$,

$$
\begin{gathered}
p_{i j}(x, y)=\left|u_{i j}(x, y)\right|^{2}, \\
U(x, x)=1, \\
U(x, y) \cdot U(y, z)=U(x, z) .
\end{gathered}
$$


REMARK 4. Notice that the reversibility of the generalized evolution $U(x, y)$, implicit in equation (28), has a purely statistical origin, stemming from the symmetric roles that two maximal observables $A(x)$ and $A(y)$ play in their mutual conditioning. Furthermore, one has

$$
u_{a b}^{*}=\frac{p_{a b}}{u_{a b}}
$$

which is the usual quantum mechanical relation.

DeFINITION 11. For each $x, y \in T$, a $\kappa$-valued transition amplitude matrix for a $\kappa$-valued bi-stochastic matrix $P(x, y)$ is a $\kappa$-valued matrix $U(x, y)$ satisfying (26).

THEOREM 6. The following assertions are equivalent:

(i1) There exists a Heisenberg algebra with center $\kappa$ satisfying conditions (i), (ii) of Theorem 5 .

(i2) For each $x, y \in T$, there exists a $\kappa$-valued transition amplitude matrix $U(x, y)$ for $P(x, y)$ such that:

$$
\begin{gathered}
U(x, x)=1, \quad \forall x \in T \\
U(x, y) \cdot U(y, z)=U(x, z), \quad x, y, z \in T .
\end{gathered}
$$

(i3) There exists a Hilbert $\kappa$-module $H$ and, for each $x \in T$, an orthonormal $\kappa$-basis $\left(a_{j}(x)\right)(j=1, \ldots, n)$ of $H$ such that the operators $A_{j}(x)$ defined by

$$
A_{j}(x) a_{k}\left(x_{0}\right)=u_{j k}\left(x, x_{0}\right) a_{j}(x), \quad \forall x_{0} \in T,
$$

do not depend on the choice of $x_{0} \in T$ and are rank one orthogonal projections.

Equations (29), (30) are a generalization of Schrödinger's evolution, which is recovered when $T=\mathbf{R}$, interpreted as time.

In the above theorem these equations appear as compatibility conditions for a set of transition probability matrices $\{P(x, y)\}$ to admit a Heisenberg algebra model.

7. Symmetries of probabilities and symmetries of amplitudes. If the index set $T$ is acted upon by a group $G$, so that amplitudes (hence probabilities too) are $G$-invariant, i.e.

$$
\begin{aligned}
& U(x, y)=U(g x, g y), \\
& P(x, y)=P(g x, g y),
\end{aligned}
$$

then one can fix an $x_{o} \in T$ and define

$$
U_{g}:=U\left(x_{o}, g x_{o}\right)
$$

Correspondingly, one has $U_{e}=1$, where $e$ is the identity in $G$ and 1 the identity operator on the $\kappa$-Hilbert space $H$. Moreover, equation (30) implies that

$$
U_{g}^{-1} U_{h}:=U\left(g x_{o}, x_{o}\right) U\left(x_{o}, h x_{o}\right)=U\left(g x_{o}, h x_{o}\right)=U_{g^{-1} h} .
$$

Thus equation (30) is also a generalization of the notion of a unitary representation. 
8. Statistical invariants for the existence of Hilbert space models. The solution of the compatibility problem given in the previous sections is not constructive: it tells us that a family of transition probabilities is canonically associated to a Schwinger algebra, i.e. (essentially) to a quantum model, if and only if one can build a family of transition amplitudes satisfying certain conditions. However it does not say how to decide, given a set of transition matrices, if these conditions are satisfied or not. Let us state this problem for the usual quantum model, i.e. in the case when $\kappa=\mathbb{C}$.

\section{Problem. Given:}

(i) a family of discrete maximal observables $\mathcal{O}:=\left\{A_{\alpha}: \alpha \in T\right\}$ ( $T$ a set),

(ii) the experimentally measurable conditional probabilities

$$
P\left(A_{\beta}=a_{j_{\beta}}^{(\beta)} \mid A_{\alpha}=a_{j_{\alpha}}^{(\alpha)}\right)=: p_{j_{\alpha}, j_{\beta}}(\beta \mid \alpha)
$$

find conditions under which there exist a complex (resp. real) Hilbert space $\mathcal{H}$ and a family of self-adjoint operators with simple spectrum

$$
\hat{A}_{\alpha}=\sum_{j} a_{j}^{(\alpha)}\left|\psi_{j}^{A_{\alpha}}\right\rangle\left\langle\psi_{j}^{A_{\alpha}}\right|
$$

such that, for any pair of observables $A_{\alpha}, A_{\beta} \in \mathcal{O}$, one has

$$
P\left(A_{\beta}=a_{j_{\beta}}^{(\beta)} \mid A_{\alpha}=a_{j_{\alpha}}^{(\alpha)}\right)=\left|\left\langle\psi_{j_{\beta}}^{A_{\beta}}, \psi_{j_{\alpha}}^{A_{\alpha}}\right\rangle\right|^{2}, \quad \forall \alpha, \beta=1, \ldots, n .
$$

First notice that a necessary condition for the existence of a Hilbert space model is that the transition probability matrices (32) are in the range of the quadratic map

$$
\left(u_{j_{\alpha}, j_{\beta}}\right) \in U n(n, \mathbb{C}) \mapsto\left(\left|u_{j_{\alpha}, j_{\beta}}\right|^{2}\right)=\left(p_{j_{\alpha}, j_{\beta}}\right) \in \operatorname{Bistoch}(n) .
$$

This is a difficult problem even in the case of 2 observables (i.e. only one bi-stochastic matrix). It is known that the map

$$
\left(u_{j_{\alpha}, j_{\beta}}\right) \in U n(n, \mathbb{C}) \mapsto\left(\left|u_{j_{\alpha}, j_{\beta}}\right|^{2}\right)
$$

is onto for $n=2$. For $n=3$, the range of this map is known but the map is not onto. No nontrivial results are available in dimensions higher than 3 . In the case $n=4$, there are computer simulations which give an idea of this range.

Now consider the case of three observables (i.e. three bi-stochastic matrices). The simplest situation is when the observables are 2 -valued, i.e. the matrices are $2 \times 2$. Thus we have the transition probabilities

$$
P\left(A=a_{\alpha} \mid B=b_{\beta}\right), \quad P\left(B=b_{\beta} \mid C=c_{\gamma}\right), \quad P\left(C=c_{\gamma} \mid A=a_{\alpha}\right) .
$$

If the three observables $A, B, C$ take only two (arbitrary) values, the associated transition probability matrices will be denoted

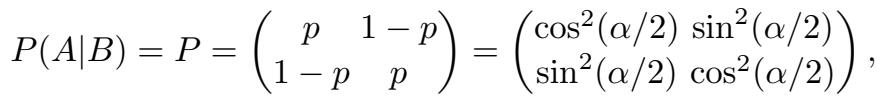

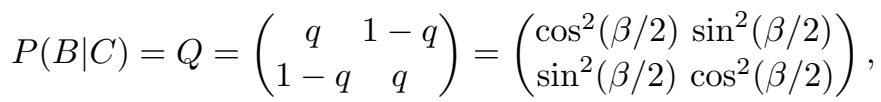




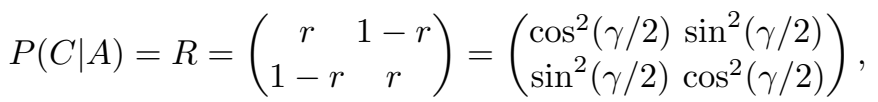

where $0<p, q, r<1,0<\alpha, \beta, \gamma<\pi$.

THEOREM 7. The following assertions are equivalent:

(i) The (experimentally measurable) transition matrices $P, Q, R$ admit a complex Hilbert space model.

(ii) The angles $\alpha, \beta, \gamma$ satisfy the inequality:

$$
\cos ^{2} \alpha+\cos ^{2} \beta+\cos ^{2} \gamma-1 \leq 2 \cos \alpha \cos \beta \cos \gamma
$$

(iii) The angles $\alpha, \beta, \gamma$ satisfy the inequality:

$$
-1 \leq \frac{\cos ^{2} \alpha / 2+\cos ^{2} \beta / 2+\cos ^{2} \gamma / 2-1}{2 \cos \alpha / 2 \cos \beta / 2 \cos \gamma / 2} \leq 1 .
$$

(iv) The transition probabilities $p, q, r$ satisfy the inequality:

$$
-1 \leq \frac{p+q+r-1}{2 \sqrt{p q r}} \leq 1
$$

THEOREM 8. The transition matrices $P, Q, R$ admit a real Hilbert space model if and only if the equality holds in the above four inequalities.

This result was proved in [AcFe81]. A simplified proof was given by Gudder and Zanghi [GuZa84] and extended to an arbitrary number of 2-valued observables in [Ac86]. Another, more elegant proof of the above theorem, based on operator trigonometry, was given by Gustafson [Gust99], [Gust00]. The elegance of the proof and the power of the general technique justify the hope that the technique itself might be applied to higher dimensions $(\geq 3)$.

9. Statistical invariants for the existence of Kolmogorov models. The compatibility problem, discussed in the previous section for the complex and real Hilbert space models, can be stated also for the Kolmogorov model.

Given the (experimentally measurable) transition probabilities (32), do there exist a probability space $(\Omega, \mathcal{F}, P)$ and $n$ measurable partitions of $\Omega$ of cardinality $n$ (= the number of distinct values of each observable),

$$
\left(A_{j}^{(\alpha)}\right), \quad j=1, \ldots, n .
$$

such that for any $\alpha, \beta \in\{1, \ldots, n\}$ one has

$$
P\left(A_{\beta}=a_{i}^{(\beta)} \mid A_{\alpha}=a_{j}^{(\alpha)}\right)=\frac{P\left(A_{i}^{(\alpha)} \cap A_{j}^{(\beta)}\right)}{P\left(A_{j}^{(\beta)}\right)} ?
$$

The necessary and sufficient conditions are reduced to a linear programming problem, i.e. to the solution of a linear system with inequality constraints, cf. [AcFe81]. Also in this case there is no general solution in a closed form, applicable to any $n$ and to any number of observables.

In the case $n=2$ and for 3 observables, one has the same transition matrices $P, Q$, $R$ as in the previous section and the result is the following [AcFe81]. 
TheOREm 9. The transition matrices $P, Q, R$, defined in the previous section, admit a Kolmogorov model if and only if

$$
|p+q-1| \leq r \leq 1-|p-q| .
$$

In this particular case (i.e. for the transition matrices $P, Q, R$ ), the following two inclusions hold:

Kolmogorov model $\subset$ complex-Hilbert-space model, real-Hilbert-space model $\subset$ complex-Hilbert-space model.

However, in general, one can only say that:

$\{$ Kolmogorov models $\} \cap\{$ complex Hilbert space models $\} \neq \emptyset$.

10. The quantum paradoxes. With the hindsight coming from almost 30 years of developments in quantum probability the origin of the so-called "quantum paradoxes" can be described as follows.

Since the very early times of quantum mechanics, physicists met sets of statistical data, coming from simply related experiments, and not satisfying some necessary conditions to be representable by means of a single Kolmogorov model (but well described by quantum mechanics, i.e. representable by means of a single complex Hilbert space model).

The first example of such data, the famous 2-slit experiment was discussed at the Solvay conference in 1928 in the debate between Bohr and Einstein and since then it remained for about 40 years the prototype example to illustrate the "mysteries of quantum mechanics" (cf. [FeLeSa66]). In modern language, the "mysteries" can be described as follows: if one applies the rules of the Kolmogorov model, then one is led to predict that the graph of the probability distributions (or correlations) will follow certain shapes. However, the graphs obtained by plotting the experimental data follow completely different patterns. According to quantum probability this means that the experimental data violate some necessary conditions for the existence of the Kolmogorov model.

In the case of the 2-slit experiment, the necessary condition, violated by the experimental data, is (a particular case of) the theorem of composite probabilities.

In 1964 Bell discovered another necessary condition for the existence of a classical probabilistic model and an example of experimental data violating it.

In this case the statistical data involved were correlations and the necessary condition was an inequality, now known as "Bell's inequality".

Before quantum probability nobody realized the common mathematical root of the two "mysteries" (i.e. the 2-slit experiment and Bell's inequality) and, in the physical literature, two mutually disjoint "explanations" for them were devised.

The 2-slit experiment gave rise to the so called "orthodox interpretation" according to which, in a superposition state with respect to a given observable (say, spin or position at a given time), this observable has only virtual values. The act of measurement marks the transition of these values from virtuality to reality. In the case of the 2-slit experiment this is translated into the statement that the passage of an electron through two separated 
slits does not correspond to two disjoint events because the electron, like a wave, "passes through both slits". Thus the theorem of composite probabilities is not applicable and this "explains" its violation.

On the contrary, Bell was convinced, and the majority of physicists for about 40 years shared this opinion, that the violation of his inequality depends on another physical fact: the mutual incompatibility of the two main contemporary physical theories: relativity and quantum mechanics. More precisely, he was convinced [Bell64] that quantum theory violates the principle of locality, which is one of the pillars of relativity theory.

The discovery of the common mathematical, in fact probabilistic, root (1980) of the 2-slit and the Bell "paradoxes" marked the birth of quantum probability not only as a mathematical theory but also as a deep revolution in our ideas concerning the laws of chance. It was also the beginning of a series of developments which, as far as the debate on the foundations of quantum mechanics is concerned, culminated in the experimental proof that there is no contradiction between locality and the Bell inequality [AcImRe01a]. The scientific solution of a historical problem not always coincides with its "sociological" solution which is subject to more complex mechanisms. However, at least one thing can be easily documented, namely that nowadays the universally accepted formulation of the Bell inequality is not the one originally given by Bell, but the quantum probabilistic formulation of [Ac81a].

In addition to the foundational questions briefly surveyed in the present paper, the birth of quantum probability was motivated by many different problems in different branches of mathematics, such as functional and stochastic analysis, operator theory, classical probability, harmonic analysis, graph theory, control and filtering theory, etc., and of physics such as quantum optics, quantum field theory, solid state physics, the theory of transport and of non equilibrium phenomena, quantum information and communication, ....

The fact that quantum probability has brought substantial innovative contributions to all of these fields is a sign of vigor of the discipline and at the same time an invitation to pursue this line of interaction and fruitful collaboration among different and often distant fields.

References. Information on Quantum Probability can be found on the web sites of:

(i) the IDAQP journal:

Infinite Dimensional Analysis, Quantum Probability \& Related Topics http://www.worldscinet.com/idaqp/idaqp.shtml

(ii) the QP-PQ series: Quantum Probability, White Noise Analysis \& Related Topics http://www.wspc.com/books/series/qqpwna_series.shtml

(iii) the European Community Research and Training Network: Quantum Probability with Applications to Physics, Information Theory and Biology

http://hyperwave.math-inf.uni-greifswald.de/algebra/qp_applications 
(iv) the web page of the association "Quantum Probability and infinite dimensional analysis":

http://www.aqpida.org

(v) A short survey of the historical development of the subject until the late 1990's is $[\mathrm{Ac00b}]$.

(vi) Some applications to concrete physical problems are described in [AcLuVo02].

\section{References}

[AcLuVo02] L. Accardi, Y. G. Lu and I. Volovich, Quantum Theory and its Stochastic Limit, Springer Verlag, 2002.

[AcImRe01a] L. Accardi, K. Imafuku and M. Regoli, On the physical meaning of the EPRchameleon experiment, Infinite Dimensional Analysis, Quantum Probability and Related Topics 5 (2002), 1-20.

[Ac00b] L. Accardi, Quantum probability: a historical survey, in: Probability on Algebraic Structures, G. Budzban, Ph. Feinsilver and A. Mukherjea (eds.), Contemporary Mathematics 30, American Mathematical Society, 2000, 145-159.

[Ac00c] L. Accardi, Quantum Probability: an introduction to some basic ideas and trends, Aportaciones Matematicas 16 (2001), 1-128.

[Ac95a] L. Accardi, Can mathematics help solving the interpretational problems of quantum theory? Il Nuovo Cimento 110 B (1995), 685-721; cf. also: Mesoscopic Physics and Fundamental Problems in Quantum Mechanics, C. Di Castro, F. Guerra and G. Jona-Lasinio (eds.), 1995.

[Ac86] L. Accardi, Foundations of Quantum Mechanics: a quantum probabilistic approach, in The Nature of Quantum Paradoxes, G. Tarozzi and A. van der Merwe (eds.), Reidel, 1988, 257-323.

[Ac82c] L. Accardi, Some trends and problems in quantum probability, in: Quantum Probability and Applications to the Quantum Theory of Irreversible Processes, L. Accardi, A. Frigerio and V. Gorini (eds.), Lecture Notes in Math. 1055, Springer, 1984, 1-19.

[Ac81a] L. Accardi, Topics in quantum probability, Phys. Rep. 77 (1981), 169-192.

[AcFe81] L. Accardi and A. Fedullo, On the statistical meaning of complex numbers in quantum theory, Lettere al Nuovo Cimento 34 (1982), 161-172.

[Bell64] J. S. Bell, On the Einstein Podolsky Rosen Paradox, Physics 1 (1964), 195-200.

[BirkvonN36] G. Birkhoff and J. von Neumann, The logic of quantum mechanics, Ann. Math. 37 (1936), 823-843.

[FeLeSa66] R. P. Feynman, R. P. Leighton and M. Sands, Lectures on Physics, III, AddisonWesley, 1966.

[Feyn51] R. P. Feynman, The concept of probability in quantum mechanics, in: Proc. 2nd Berkeley Symp. Math. Stat. Prob., University of California Press, Berkeley, 1951, $533-541$.

[Gleas57] A. M. Gleason, Measures on closed subspaces of Hilbert space, J. Math. Mech. 6 (1957), 885-893.

[GuZa84] S. Gudder and N. Zanghi, Probability models, Nuovo Cim. B 79 (1984), 291-301. 
[Gust99] K. Gustafson, The trigonometry of quantum probabilities, in: L. Accardi et al. (eds.), Trends in Contemporary Infinite Dimensional Analysis and Quantum Probability, Scuola di Studi sull'Asia Orientale, Natural and Mathematical Sciences series N. 3, Kyoto (1999) (Hida Festschrift).

[Gust00] K. Gustafson, Quantum trigonometry, IDA-QP (2000), 33-52.

[HaaKa64] R. Haag and D. Kastler, An algebraic approach to quantum field theory, J. Math. Phys. 5 (1964), 848-861.

[Hei27] W. Heisenberg, Über den anschaulichen Inhalt der quantentheoretischen Kinematik und Mechanik, Z. für Phys. 40 (1927), 172-198.

[HvNN27] D. Hilbert, J. von Neumann and P. Nordheim, Über die Grundlagen der Quantenmechanik, Math. Annal. 98 (1927), 1-30; also in: J. von Neumann, Coll. Works, Pergamon Press, vol. I, 104-133.

[Jau68] J. M. Jauch, Foundations of Quantum Mechanics, Addison-Wesley, 1968.

[Kolmo33] A. N. Kolmogoroff, Grundbegriffe der Wahrscheinlichkeitsrechnung, Springer Verlag, Berlin, 1933; reprinted: Foundations of the Probability Theory, Chelsea Publ. Comp., New York, 1956.

[Mackey76] G. W. Mackey, Mathematical Foundations of Quantum Mechanics, AddisonWesley, 1976.

[Paszk85] A. Paszkiewicz, Measures on projections in $W^{*}$-factors, Journal of Functional Analysis 62 (1985), 87-117.

[Piron76] C. Piron, Foundations of Quantum Physics, Addison-Wesley, 1976.

[RedStöl01] M. Redei and M. Stöltzner (eds.), John von Neumann and the Foundations of Quantum Physics, Kluwer, 2001.

[Segal47] I. E. Segal, Postulates for general quantum mechanics, Annals of Mathematics 48 (1947), 930-948.

[Varadar68] V. S. Varadarajan, Geometry of Quantum Theory, van Nostrand, 1968. 
